\title{
La categorización de las palabras compuestas en español ${ }^{1}$
}

\section{Categorizing compounds in Spanish}

\author{
BÁRBARA MARQUETA \\ Universidad de Zaragoza, Facultad de Filosofía y Letras. España. \\ Correo electrónico: marqueta@unizar.es
}

El objetivo fundamental de este artículo es esbozar una propuesta de categorización de los compuestos del español en torno a rasgos sintácticos. Tras una breve presentación de las teorías semánticas y formales de categorización, ejemplificaremos aquellas proyecciones funcionales categoriales (p.e. género) cuya ausencia o presencia determina las particularidades semánticas y distribucionales de cada esquema compositivo. Trataremos de demostrar, por un lado, que existe especificidad categorial en el interior de los compuestos, aunque dicha especificidad categorial no sea morfofonológicamente manifiesta. Por otro lado, defenderemos la existencia de una mayor cantidad de estructura funcional y relaciones gramaticales de la que tradicionalmente se contempla, especialmente en lo que respecta a los modificadores compositivos.

Palabras claves: categoría, compuesto, sintaxis, morfología.

The main aim of this paper is to present a proposal of compound categorization from syntactic features. After a brief presentation of both semantic and formal approaches to categorization, we will exemplify the functional projections (i.e. gender) whose presence or absence determines the semantic and distributional characteristics of each compound pattern. We will try to show that, on the one hand, there exists category-specific behaviour inside compounds, although the referred specificity is no necessarily expressed morphologically. On the other hand, we will maintain that there exists a bigger amount of functional structure and grammatical relationships than traditionally observed, especially regarding compound modifiers.

Key words: category, compound, syntax, morphology.

\footnotetext{
${ }^{1}$ Esta investigación se ha desarrollado en el proyecto "Conceptos, estructuras y sonidos: una aproximación pluralista a los ingredientes esenciales del lenguaje humano”, en el marco del Programa Estatal de Fomento de la Investigación Científica y Técnica de Excelencia (FECYT). Asimismo, Bárbara Marqueta ha contado con la financiación del Ministerio de Educación, Cultura y Deporte (FPU 14/01500).
} 


\section{INTRODUCCIÓN}

Si acudimos a la definición del término categoría que ofrece el $D R A E$, las acepciones vinculadas a la especialidad gramatical aluden específicamente a las categorías gramaticales o clases de palabras. La realidad de los estudios de la lingüística contemporánea, sin embargo, es mucho más heterogénea, y quizá por ello muchos de los conceptos vinculados a la disciplina de la Filosofía, como las categorías de la lógica aristotélica, no resultan extraños en los estudios lingüísticos actuales sobre la cuestión categorial.

La aproximación multidisciplinar a la delimitación categorial ha sido bienvenida por varias razones, de las que cabe destacar fundamentalmente dos: en primer lugar, porque, pese a que la identificación y delimitación del repertorio de categorías gramaticales constituye uno de los temas más persistentes de los estudios de las lenguas, todavía no ha sido resuelto de manera satisfactoria. En segundo lugar, porque el estudio de las categorías gramaticales en lenguas totalmente desconocidas para la tradición occidental de análisis ha puesto en tela de juicio algunos de los presupuestos tradicionales sobre las categorías.

La diversidad lingüística, por tanto, tanto aquella que se puede encontrar dentro de una misma lengua como la existente de manera universal en las lenguas, supone un reto para las aproximaciones gramaticales a la noción de categoría. El tema concreto de esta contribución, la identificación de categorías en las palabras compuestas del español, está vinculado al primer tipo de diversidad, dado que las propiedades de las categorías en los compuestos son diferentes a las propiedades de las categorías en la oración, aunque uno de los objetivos de la contribución será demostrar que estas diferencias no son inherentes a la condición de "palabra" del compuesto, puesto que se pueden explicar atendiendo a sus propiedades estructurales. Por ejemplo, está claro que el verbo en el interior de compuestos como lavaplatos no puede flexionar tiempo *lavabaplatos, frente a lo que ocurre en la oración Él lavaba platos.

El presente trabajo se estructura de la manera siguiente. En el segundo apartado, repasaremos brevemente los principales tipos de aproximaciones a la identificación de categorías en la lingüística actual. En el tercer apartado, presentaremos los denominados proyectos cartográficos de las categorías lingüísticas, destacando sus ventajas para abordar la cuestión de la diversidad lingüística que esbozábamos en los párrafos anteriores. En el cuarto apartado presentaremos algunas de las propuestas más influyentes sobre categorización de compuestos, comentando brevemente sus principales ventajas e inconvenientes.

Los apartados quinto, sexto y séptimo constituyen el núcleo central de este trabajo. En ellos se ejemplifica la implementación de un análisis cartográfico a los compuestos que contienen sustantivos, adjetivos y verbos, respectivamente. La hipótesis fundamental que defenderemos, de manera general, es que la aproximación cartográfica a las categorías ayuda a capturar la vieja intuición de que la composición es una microsintaxis (Benveniste 1966).

Concretamente, en el apartado quinto, demostraremos cómo la mayor o menor presencia de elementos funcionales es la fuente de variación morfofonológica en los compuestos con sustantivos. Los compuestos con sustantivos en (1) están ordenados, 
de izquierda a derecha, en función de la menor o mayor complejidad funcional de los sustantivos en cursiva.

\section{(1) drogodependencia <lavaplatos < camiones cisterna}

En la segunda mitad del apartado quinto, así como el apartado sexto, destacaremos la necesidad de ampliar la nómina de relaciones gramaticales que se establecen en el interior de un compuesto, pues los estudios de composición normalmente se limitan a identificar relaciones de coordinación, subordinación y modificación. Podremos identificar diferencias sistemáticas entre compuestos a expensas de si contienen predicados, adjuntos o complementos restrictivos. El último de los contrastes se recoge en (2).

(2) sietemesino (siete es adjunto) vs videoaficionado (video es complemento)

Finalmente, en el apartado sexto, se defenderá que la estructura predicativa de los compuestos verbonominales (abrelatas) es más compleja que la de los llamados compuestos sintéticos equivalentes en una lengua como el inglés (can opener). Ello determina diferencias interpretativas sistemáticas, pero también fonológicas: mientras que la proyección del verbo en espańol se identifica por una forma flexionada de $3^{a}$ persona del singular (abre) la proyección verbal del inglés es identificada por un tema de infinitivo (open).

\section{IDENTIFICACIÓN DE CATEGORÍAS EN LA TEORÍA LINGÜÍSTICA}

Las grandes teorías sobre la categorización lingüística dan prominencia a uno de los planos de la categorización, bien sea el semántico, bien el formal.

Así, para aquellos autores que consideran la categorización un fenómeno esencialmente semántico o pragmático, existe una relación estrecha entre categorías lingüísticas y sus funciones discursivas, p.e., entre el verbo y la predicación (Croft 1984) o entre las categorías y los conceptos que expresan, p.e., entre verbo y cambio (Givon 1979). De esta manera, podemos identificar miembros prototípicos de cada categoría, como lo es casa de la categoría sustantivo, pero también miembros periféricos como el sustantivo manutención.

Estos modelos constatan la dificultad de caracterizar las categorías lingüísticas de manera discreta. La relativización de la identificación de categorías vinculada al concepto de prototipicidad semántica/funcional no soluciona los problemas de categorización, sin embargo, pues dicho concepto, el de prototipicidad, siempre es dependiente del plano o perspectiva desde el cual se aplica. Esto es, algo es prototípico o no prototípico siempre respecto a algo. Manutención y extender(se) son miembros poco prototípicos de sus categorías respectivas en cuanto a su significado (el nombre denota un proceso y el verbo un estado), pero ninguna propiedad formal está ausente (el nombre manutención tiene género y el verbo 
extenderse, tiempo). Por el contrario, miembros prototípicos de la categoría verbal respecto a su significación, como llover y acontecer, sí tienen propiedades formales ausentes: * Cuando los cielos llovieran, yo acontecería.

Así, sería deseable que la prototipicidad no fuese patrimonio de ninguna teoría sobre la categorización lingüística, ni necesariamente incompatible con la delimitación discreta de las categorías. Aplicando la célebre sentencia, nos atreveríamos a afirmar que "la prototipicidad está en el ojo de quien mira”.

Entre aquellos autores que consideran la categorización lingüística un fenómeno esencialmente formal se han propuesto diferentes sistemas que tratan de ofrecer, con mayor o menor éxito, una identificación categorial en torno a propiedades estructurales. Chomsky (1970: 208) propone un sistema de rasgos en el que, por ejemplo, las categorías $+\mathrm{V}$ (verbos y preposiciones) son capaces de asignar directamente caso a sus complementos (entró la mesa, entre las ramas) a diferencia de sustantivos y adjetivos, que lo hacen a través de una preposición (adentramiento en la tierra, familiarizado con el problema).

Baker (2003), tras analizar una notable muestra de lenguas, propone un sistema de identificación categorial en el que las propiedades formales y semánticas de las categorías son interdependientes. Así, por ejemplo, los verbos son la categoría predicativa por excelencia (propiedad semántica) y siempre proyectan una posición sintáctica para su argumento interno (propiedad formal). En Hinzen (2008) el significado categorial depende absolutamente de las propiedades formales de la estructura, es decir, de los tipos de referencia construidos: un Sintagma Determinante siempre construye una referencia a individuos, un Sintagma Complementante una referencia a proposiciones, etc.

Finalmente, destacan por su divulgación actual los llamados modelos neoconstruccionistas. En estos modelos las categorías no forman parte de la información del léxico, como en la mayoría de los modelos. La categorización es una operación sintáctica que se produce cuando una raíz se ensambla con diferentes tipos de categorías funcionales (Borer 2013).

La nota común de los modelos formales es que aspiran a ofrecer una delimitación discreta de las categorías. Cada uno cuenta con sus particulares limitaciones, sin embargo. Baker (2003: 275) acepta como premisa que su modelo no es aplicable por debajo del nivel de palabra. Para el autor, green en green house 'casa verde' posee categoría, no así en greenhouse 'invernadero', pese a que ninguno de sus criterios sintácticos para identificar la categoría adjetivo se incumple en el compuesto. El neoconstruccionismo asume un alto grado de sobregeneración. De algunas versiones se desprende que, por defecto, cualquier raíz puede adscribirse a dos o tres categorías distintas, sistema que es mucho más plausible en unas lenguas que en otras. La adscripción categorial depende de unos elementos funcionales que, en muchos casos, no tienen manifestación morfológica y, en otros casos, ofrecen la misma problemática de adscripción categorial que los elementos léxicos (p.e. ¿a qué categoría pertenece $u n$ ?).

En este trabajo se adopta una teoría formal sobre la categorización que combina aspectos del modelo de Baker y de los modelos neoconstruccionistas. Este modelo nos 
debe permitir afirmar que parabrisas contiene dos categorías (un verbo y un sustantivo), sin renunciar a considerar verbo a para porque parte de sus propiedades como verbo estén ausentes (esto es, no lo consideraremos una raíz acategorial). Paralelamente, al no asumir un modelo lexicista, no nos vemos en la obligación de tener que explicar por qué, estando para léxicamente (inherentemente) especificado como verbo, carece de algunas de las propiedades de los verbos de una lengua. Para nosotros esas propiedades ausentes están proporcionadas por la estructura, al igual que en los modelos neoconstruccionistas. Nuestras principales asunciones acerca de la identificación de categorías son las siguientes:

- La ausencia de determinadas propiedades (p.e. las denominadas flexivas) no puede determinar necesariamente la ausencia de una categoría.

- La presencia de determinadas propiedades (como vocales de tema en nombres y adjetivos y vocales temáticas en verbos) excluye la posibilidad de identificar cierta entradas léxicas como miembros de varias categorías.

- La presencia de una determinada propiedad (p.e. género) implica la presencia de otras propiedades (número).

\section{LOS PROYECTOS CARTOGRÁFICOS}

Normalmente se considera Rizzi (1997) el trabajo pionero de las cartografías. En los modelos cartográficos, lo que denominamos, por ejemplo, nombre (o sintagma nominal) está integrado por la unión de un elemento del léxico con un conjunto de categorías funcionales con semántica y/o función específica (género, número, etc.). Una categoría gramatical queda definida, pues, por un conjunto jerarquizado de rasgos formales.

La jerarquización de los rasgos formales es esencial para explicar las diferencias entre los modelos cartográficos y cualquier otro tipo de sistema que ofrece una descripción lingüística en torno a rasgos. Por ejemplo, la caracterización de un fonema a través de rasgos no es necesariamente ${ }^{2}$ jerárquica: la caracterización de un fonema con un punto de articulación bilabial, un modo de articulación oclusivo y sin vibración en las cuerdas vocales (sordo) nos conduce al sonido $/ \mathrm{p} /$ independientemente de si bilabial o sordo va antes o después en la caracterización.

Los rasgos que caracterizan una categoría gramatical sí son necesariamente jerárquicos. Si dichos rasgos aparecen en distinto orden, el resultado puede ser desde agramatical hasta una categoría diferente. Es por eso precisamente que hablamos de morfemas derivativos y no de fonemas derivativos.

La jerarquización de rasgos en una estructura sintáctica es completamente indiferente a su manifestación morfofonológica, o lo que es lo mismo, las categorías están jerárquicamente organizadas tanto en la sintaxis oracional como en la llamada sintaxis de

\footnotetext{
${ }^{2}$ Tenemos en cuenta que la presencia de una vocal implica rasgo sonoro u otras dependencias.
} 
la palabra. Como se observa en (3), una jerarquización correcta da resultados gramaticales tanto si el resultado morfofonológico es una palabra como si es un sintagma, mientras que una jerarquización imposible da resultados agramaticales en cualquiera de los dos casos.

(3) Género $>$ clasificador $>$ Grado $>$ Adjetivo $^{3}$

$\begin{array}{llll}\text { la } & \text { ía } & \text { mejor } & \text { 'la mejoría' } \\ \text { la } & 0 & \text { más grande } & \text { 'la más grande' }\end{array}$

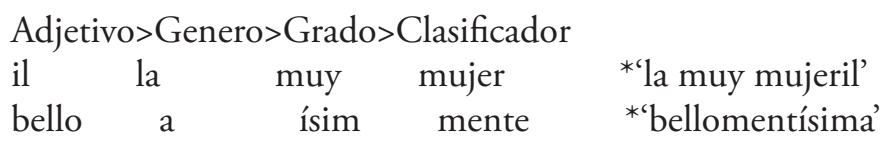

Otra cuestión relevante de cara a la elaboración de cartografías atañe a la obligatoriedad u opcionalidad de los rasgos. Se discute si todos los rasgos propuestos deben proyectarse universalmente, conformar matrices (Bobaljik y Thráinsson 1998) o ser opcionales (Starke 2004). La reflexión normalmente se orienta hacia el problema de la variación interlingüística, al que referíamos en la introducción. En lo que respecta a la variación dentro de la propia lengua, y si consideramos que la estructura de las palabras forma parte de esta variación (en otras palabras, si consideramos que las palabras se forman con las mismas categorías gramaticales que los sintagmas y oraciones de la lengua), la respuesta a la cuestión de la opcionalidad de los rasgos es clara: sí debe haber proyecciones funcionales prescindibles u opcionales en la cartografía de una categoría.

\section{Categorización de Compuestos}

El procedimiento clasificatorio de compuestos más arraigado, que se ejemplifica en (4), consiste en describir patrones de combinatoria en los que cada uno de los constituyentes de un compuesto recibe una etiqueta categorial, mientras que el conjunto recibe otra que puede coincidir (compuesto endocéntrico) o no (compuesto exocéntrico) con la del constituyente que se estipula como núcleo. ${ }^{4}$ La convención no impide que en cada línea teórica de análisis se perciba una concepción muy diferenciada de la naturaleza de las categorías de los constituyentes.

\footnotetext{
${ }^{3}$ Los elementos a la derecha en la representación son los que se sitúan en el nivel inferior de la jerarquía funcional. Estos se van ensamblando progresivamente con los elementos situados a su izquierda. Las glosas reflejan la asunción típica sobre la linearización de elementos morfológicos (núcleos a la derecha de sus complementos) frente a la linearización de elementos sintácticos (núcleos a la izquierda).

${ }^{4}$ Esta clasificación es transversal a diferentes marcos teóricos, podemos encontrarla en los capítulos dedicados a la composición en las gramáticas descriptivas (Val Álvaro 1999), y en distintos monográficos sobre el tema (Moyna 2011).
} 
(4) $[\mathrm{N}+\mathrm{N}]_{\mathrm{N}}$ camión cisterna, aromaterapia

$[\mathrm{V}+\mathrm{N}]_{\mathrm{N}}$ limpiabotas, soplagaitas

$[\operatorname{Adj}+\mathrm{N}]_{\mathrm{N}}$ altavoz, malasombra

$[\mathrm{P}+\mathrm{N}]_{\mathrm{N}}$ sinsabor, antebrazo

Entre los modelos de categorización más influyentes se encuentran los de "filtrado de categoría". En estos modelos, uno de los constituyentes del compuesto (normalmente el que está situado a la derecha o más externo) posee la categoría que se filtra al compuesto en su totalidad (Lieber 1992). La repercusión más notable de esta tradición analítica es la consideración de compuestos como lavaplatos como la versión exocéntrica o derivada (mediante un morfema cero) del compuesto inglés dish-washer (apartado 7). Tal consideración, a nuestro parecer, hace que el porcentaje de compuestos exocéntricos en las lenguas romances incremente injustificadamente hasta el 35\%, casi doblando la cifra de compuestos exocéntricos de las lenguas del Este asiático (Scalise et al. 2009: 5).

Los modelos neoconstruccionistas se decantan por un sistema de categorización de raíces (Harley 2009). En estos sistemas, la palabra compuesta no está categorizada hasta que uno de los constituyentes se ensambla con una categoría funcional. La ausencia de ciertas categorías funcionales en la estructura interna de los compuestos justifica su tratamiento como estructuras incorporantes. Estas propuestas solucionan alguno de los problemas de los modelos de filtrado, como la exocentricidad morfológica que ejemplifica el parabrisas, compuesto en el cual el género de brisas no concuerda con el del artículo el. ${ }^{5}$ Esta aproximación, sin embargo, resulta problemática para aquellos casos en los que encontramos especificidad categorial en el interior de una palabra compuesta (apartado 6).

Finalmente, los modelos que mayor difusión han tenido en las últimas décadas son los que contemplan algún tipo de separación entre los componentes morfológico y sintáctico. Se considera que la categoría es una propiedad gramatical común a unidades morfológicas y sintácticas, mientras que otras propiedades, como la concordancia, solo operan con las unidades sintácticas (Rainer y Varela 1992; Ackema y Neeleman 2004). En estos modelos se distinguen compuestos formados en el componente morfológico como lavaplatos de compuestos formados en la sintaxis y reanalizados como objetos léxicos como llave inglesa.

Nuestra aproximación sintáctica a la composición no contempla la separación entre un componente formador de palabras y otro formador de oraciones dado que, desde una aproximación cartográfica, es posible vincular la ausencia de propiedades (como concordancia) a la ausencia de proyecciones funcionales en la estructura que denominamos compuesto. Todos los compuestos estarían formados en la sintaxis y unidades como llave inglesa no serían en ningún caso compuestos, sino sintagmas provistos de significado idiomático, como lo es enterrar el hacha de guerra.

5 Aunque este tipo de divergencias entre la flexión del conjunto y la flexión del núcleo se producen frecuentemente con palabras simples (un manitas, el coletas) y nadie habla de exocentricidad morfológica en estos casos. 


\section{LA CARTOGRAFÍA DE LA CATEgoría SUSTANTIVO}

En este apartado vamos a analizar diferentes tipos de compuestos integrados por sustantivos (camión cisterna, actrices estrella, limpiabotas, drogodependencia, etc.). Los sustantivos presentan evidentes diferencias morfológicas en estos compuestos (compárese drogo con actrices), que en este capítulo vamos a relacionar con la presencia de mayor o menor estructura funcional en el compuesto. Es importante puntualizar que en este trabajo un compuesto no está formado únicamente por la unión de dos lexemas, pues una mayor o menor cantidad de estructura sintáctica media el ensamble de los mismos. Dicha estructura, en un modelo cartográfico, se representa como en (5):

(5) Proyección funcional de un sustantivo en un compuesto: Determinante $>\mathrm{N}$ úmero>Género>Clasificador

Clasificador constituye el nivel en el cual el sustantivo se adscribe a un género o declinación de manera inherente. Dicha proyección se encarga de codificar aspectos semánticos como el carácter contable del sustantivo (Borer 2005a). La proyección inmediatamente superior, que es Género, constituye el nivel donde un sustantivo establece relaciones de concordancia con otros miembros de su mismo sintagma. La necesidad de diferenciar esas proyecciones se argumenta en Fábregas y Pérez Jiménez (2008). Aunque normalmente clasificador y género coinciden morfológicamente en los sintagmas y por ello son difíciles de diferenciar, encontramos ejemplos, como los de (6), donde su existencia independiente sí se puede observar (p.e., cuando encontramos asimetrías entre el género (mejor dicho, el clasificador) del nombre y el género de un indefinido, resulta obvio el clasificador nominal no necesariamente determina la concordancia en el sintagma).

(6) Soy toda oídos, un poco de leche (frente a una poca de leche)

Vamos a encontrar evidencia adicional para diferenciar estas proyecciones en los compuestos. Una notable cantidad de sustantivos en los compuestos del español proyectan hasta el nivel de clasificador, pero nunca alcanzan el nivel de género. Es el caso de los sustantivos cerrados por vocales de tema (7a), antiguas marcas de caso (7b) u otras categorías funcionales (7c).

(7) a. videoaficionado, vasodilatador

b. canceriforme, drogodependiente

c. carricoche, pelirroja

Las proyecciones de sustantivos al nivel de clasificador se caracterizan por las siguientes propiedades (o, mejor dicho, ausencia de ellas). No permiten concordancia (8a). No codifican propiedades semánticas relevantes vinculadas a la referencia (8b). La estructura funcional es insuficiente para delimitar el tipo de modificación que representan (8c). 
(8) a. * dos-pl. vasos-m.pl-dilatadores-m.pl

b. un drogodependiente lo puede ser a las drogas en general o a un tipo de droga en particular

c. aromaterapia ¿terapia aromática o terapia a base de aromas? eurodiputado ¿diputado europeo o representante en la Unión Europea?

El siguiente tipo de proyección de un sustantivo que encontramos en compuestos incluye, además de clasificador, las proyecciones de género y número (la presencia de género implica la de número de manera universal en las lenguas, como ya recogió Greenberg en uno de sus famosos universales).

La proyección de número está relacionada con la posibilidad de encontrar un plural morfológico en el compuesto, aunque es necesario distinguir entre una proyección léxica y una funcional de plural, la última de las cuales se manifestaría a través de la concordancia de número. En los compuestos de (9) encontramos únicamente la proyección léxica del plural, dado que el sustantivo en el compuesto no establece una relación de concordancia (9b). ${ }^{6}$ No obstante, la presencia de las proyecciones de género y de número determina la posibilidad de codificar en el compuesto aspectos semánticamente interpretables de la referencia del sustantivo, a diferencia de lo que hacía la proyección de clasificador en (8).

Ya ha sido observado con anterioridad que el plural de manchas o fuegos en los compuestos de (9) se acomoda a un tipo de predicación genérica o habitual (Varela 1990). Este plural semántico hace que la participación de un objeto en el evento sea relevante en virtud de la iteración (9c). Además, coerciona la interpretación de los nombres de masa y los individualiza, convirtiéndolos en contables (9d).

(9) a. quitamanchas, cortafuegos

b. $e$-m.sing quita-manchas-fem.pl

c. ¿Es útil un friegasuelos de un solo uso y para un único cuarto?

d. paraguas (aguas de distintas lluvias); cortafuegos (de distintos incendios).

Finalmente, en un compuesto podemos encontrar la proyección completa de un sustantivo, esto es, la que incluye no solo clasificador, género y número, sino también determinante (10). Esta proyección añade respecto a las anteriores la obligatoria concordancia en género y número del sustantivo en posición interna (10b).

(10) a. el pez globo, las actrices estrella

b. los camiones cisterna/*las camión cisternas

\footnotetext{
${ }^{6}$ Excepcionalmente, cuando el sustantivo aparece en singular, como en el girasol, sí hay concordancia (los girasoles y no ${ }^{*}$ los girasol). En este y otros casos similares podríamos aducir que la proyección del sustantivo no sería al nivel de Número, sino inferior.
} 
En (11) hacemos un resumen de los tipos de proyección de sustantivo junto con algunos ejemplos representativos.

(11) Proyección funcional de $\mathrm{N}$ en compuestos:
a. clasificador
número $>$ género $>$ clasificador
determinante>número >género>clasificador
(los) vasodilatadores
(un) posavasos
(los) vasos estrella

Si dentro de un compuesto existen distintos niveles estructurales, como hemos mantenido hasta el momento, lo esperable es que podamos encontrar diferencias semánticas en función de la posición en la que se produce el ensamble entre los dos elementos léxicos del compuesto. Ello ocurre en la sintaxis oracional, como demuestra Cinque (2014), que sistematiza las diferencias interpretativas entre ensamblar adjetivos como modificadores directos o adjuntos de un nombre (un buen hombre) y ensamblarlos como predicados (un hombre bueno). Es evidente que las expresiones no significan lo mismo en español. A continuación, trataremos de demostrar que esta misma diferencia de posición de ensamble es la que nos permite interpretar de manera diferente los modificadores de las dos series de compuestos de (12).

(12) a. coche bomba, pez espada, camión cisterna

b. actriz estrella, aerolíneas modelo, madre coraje

Es una observación antigua que los modificadores en las construcciones de (12b) parecen más próximos a la categoría adjetivo que los de (12a). Autores como Val Álvaro (1999: 4787) han considerado preferible, por ello, tratar la serie de (12b) como aposiciones y no como compuestos; García Page (2011) desarrolla esta última idea. Siguiendo a Fábregas (2005), consideramos que ambas series pueden asimilarse a las aposiciones de tipo especificativo, ${ }^{7}$ si bien hay que notar que únicamente los modificadores de la serie de (12b) aparecen con frecuencia en aposiciones de tipo enfático (13).

(13) ¡Qué prodigio de hijo tienes: es un modelo de conducta y una estrella del baloncesto!

A continuación, vamos a enumerar las razones por las cuales consideramos que todos los compuestos de (12), y no solo los de (12b), deben ser tratados como construcciones sintácticas. En primer lugar, un mismo ejemplo puede crear contextos de

\footnotetext{
${ }^{7}$ Cabe puntualizar que, en nuestra aproximación, no existe incompatibilidad alguna en considerar que algunos tipos de aposiciones (construcciones en las que un nombre modifica directamente a otro nombre) puedan considerarse compuestos. Cuáles sí y cuáles no es un aspecto que no desarrollaremos aquí por motivos de espacio.
} 
ambigüedad estructural (14), hecho que, en mi opinión, es más difícil de explicar si una de las construcciones se construye en la morfología y la otra lo hace en la sintaxis.

(14) El pez estrella del zoo

Estrella como adjunto: El único pez de su especie en el zoo Estrella como predicado: El pez más famoso del zoo

En segundo lugar, los modificadores de (12) se comportan como los modificadores sintácticos, en el sentido de que ocupan diferentes posiciones jerárquicas, motivo por el cual su orden no es intercambiable (15).

(15) Predicado $<$ Adjunto $<$ Nombre El pez globo estrella

Identificamos, entre los peces globo, al más famoso p.e. Flounder. Adjunto $<$ Predicado $<$ Nombre. *El pez estrella globo

*Identificamos, entre los peces más famosos, al de la especie globo.

Los autores que consideran compuestos a los ejemplos de (12a) se basan normalmente en la mayor idiomaticidad de estas construcciones, frente a la composicionalidad de las de (12b). Sin embargo, en una propuesta como la de Cinque, la posición de adjunción del modificador determinaría la posible interpretación idiomática del modificador (no daríamos por hecho que los objetos morfológicos son idiomáticos y los sintácticos no lo son). Así, los modificadores de los compuestos se comportarían como los de la sintaxis oracional, donde un modificador adjunto puede configurar significados idiomáticos con su núcleo (16a), pero un predicado no puede dar lugar a significados idiomáticos con su núcleo/sujeto (16b).

(16) a. Han trasladado la mesa redonda a otra sala. (idiomático o no)

b. Han trasladado la mesa que es redonda a otra sala. (composicional)

También es correcta la observación de que, en la serie (12a), la interpretación semántica del modificador depende del núcleo (17). Sin embargo, en la serie (12b) el significado del modificador es independiente del significado del núcleo: es igual de estrella la actriz estrella que el cenicero estrella.

(17) pájaro carpintero (carpintero es metafórico)

bombero carpintero (carpintero no metafórico)

Los modificadores de la serie (12b) se asimilan con facilidad a los nombres usados predicativamente (18a); esto no ocurre con los de (12a), puesto que no son nombres predicativos (18b). 
(18) a. María es la alcaldesa; Juan es su alumno

Penélope Cruz es la estrella; Mi sobrina es un prodigio.

b. camion cisterna $={ }^{*}$ 'El camión es un cisterna'.

coche bomba $={ }^{*} \mathrm{El}$ coche es la bomba ${ }^{8}$

Basamos nuestro análisis de las diferencias entre las construcciones de (12) en la posición de adjunción de sus modificadores. La posición de adjunción pretende explicar la existencia de ambigüedades estructurales (14), así como las diferencias de orden (15) y de integración semántica existentes entre ambas series (16-17).

Teniendo en cuenta la estructura funcional de un sintagma nominal que presentábamos en (5) -Determinante>Número $>$ Género $>$ Clasificador-, consideramos que los modificadores de la serie de (12a) se proyectan como adjuntos de Clasificador. Cuando los modificadores se proyectan en dicha posición, contribuyen a establecer la referencia del núcleo, lo que explica su comportamiento en (16) y (17). Proyectamos los modificadores de la serie de (12b) como predicados por debajo de Determinante, p.e., al nivel de Número. La razón es que el modificador de un compuesto nunca proyecta determinante en el compuesto ( ${ }^{*}$ actriz la estrella). En este nivel más alto de la proyección, el sustantivo nuclear ya ha establecido su referencia, explicándose su composicionalidad y autonomía semántica respecto al modificador predicativo, así como su relación con estructuras predicativas oracionales (13) y (18).

\section{LA CATEGORÍA DE LOS MODIFICADORES IMPORTA: EL CASO DE LOS COMPUESTOS ATRIBUTIVOS}

En este apartado, que se centra en los compuestos de núcleo adjetivo, defenderemos la idea de que, pese a la existencia de un alto grado de neutralización morfológica, en la posición interna de un compuesto se pueden codificar relaciones de complementación diferentes a las de modificación.

Tomando nuevamente la referencia de Cinque (2014), establecemos una correlacion entre las dos clases de adjetivos que el autor propone (modificadores y predicados) y el tipo de relación atributiva que establecen en un compuesto, la que denominaremos interna o entre los propios constituyentes del compuesto, y la externa, que se establece entre el núcleo del compuesto y un elemento ajeno al mismo. En (19-22) se ejemplifican en español e inglés las cuatro combinaciones posibles.

(19) Modificadores que realizan atribución interna (p.e. mal se atribuye a humor)9: mal y uno en malhumorado o unilateral

bad y one en bad-tempered o one-sided

\footnotetext{
${ }^{8}$ Esto sería la glosa para un compuesto de la serie de (12b) como noticia bomba.

${ }^{9}$ Tenemos en cuenta que, por ejemplo, humorado no es una palabra del español, pero este hecho no es pertinente para nuestra propuesta.
} 
(20) Modificadores que realizan atribución externa ( humorado atribuido a Juan ${ }^{10}$ : humorado y lateral en malhumorado o unilateral tempered y sided en bad-tempered o one-sided

(21) Predicados que realizan atribución interna (condensada atribuido a leche): condensada y láctea en leche condensada o vía láctea condensed y milky en condensed milk o milky way

(22) Predicados que realizan atribución externa (dependiente atribuido a Juan): Dependiente y rojo en drogodependiente y pelirrojo Blind 'ciego' y sick 'enfermo' en color blind 'daltónico' y home-sick 'melancólico'

En (23) mostramos que los contrastes señalados en (19-22) son relevantes en la sintaxis oracional de cara a la selección del auxiliar o el orden relativo de los constituyentes. Los contrastes serán también relevantes en el ámbito de la composición.

(23) Juan tiene mal humor/*Juan es mal humor * Juan tiene malhumoradolJuan es malhumorado Esta leche es condensada/*Vendió condensada leche * Juan es droga dependientelJuan es dependiente de las drogas

Aunque los compuestos con modificadores (19-20) son mucho más productivos en inglés que en español, el tránsito de la atribución interna a la externa es similar en ambas lenguas, pues se obtiene mediante sufijos.

Siendo la anteposición al sustantivo la posición del adjetivo no marcada en inglés (aparecen antepuestos tanto modificadores como predicados) las diferencias entre (19) y (21) en inglés no son morfofonológicamente manifiestas. Sin embargo, siendo la antepuesta una posición marcada para el adjetivo en español (en dicha posición no aparecen predicados), comprobamos que las diferencias en nuestra lengua entre modificadores (19) y predicados (21) sí son evidentes en lo que respecta a la atribución interna.

Los adjetivos pospuestos al nombre son en inglés predicados de atribución externa de manera no ambigua (22), pues un modificador nunca aparece pospuesto al sustantivo en esta lengua. Sin embargo, los casos del español pueden (y ocasionalmente suelen) interpretarse como instancias de atribución interna. Concretamente, esto ocurre en los estudios de referencia sobre el tipo de pelirrojo.

\footnotetext{
${ }^{10}$ Nos decantamos por considerar que el proceso de modificación de (19) se produce antes que el de atribución externa en (20). No obstante, identificamos únicamente el nombre adjetivado como el elemento atribuido al sujeto, dado que la atribución es de tipo posesivo (se dice de Juan que tiene humor o de algo que tiene lados).
} 
Uno de los análisis más extendidos asume que existe una relación predicativa entre pelo y rojo (Rainer y Varela 1992). De este modo, pelirrojo tendría que reagruparse con los compuestos de (21). El principal problema es que pelirrojo no comparte una sola propiedad, semántica, morfológica o fonológica, con leche condensada.

La otra opción consiste en considerar pelirrojo la variante sin sufijo -o con afijo medial- de los compuestos ingleses de (20) como red-haired (Gil Laforga 2014). Dado que todos los adjetivos son analizados como predicados en esta propuesta, formarían también parte de un grupo común con (19). Sin embargo, el hecho de que un compuesto del inglés como home-sick presente más propiedades morfológicas (ausencia de morfema derivativo, posición interna del sustantivo) en común con pelirrojo que red-haired nos puede conducir a replantearnos las ventajas de una unificación de patrones en torno a (20) cuya variación (p.e., posición del afijo) se considera exclusivamente morfológica.

En nuestro análisis planteamos las diferencias entre compuestos con núcleos adjetivos en torno a la categoría del no-núcleo o elemento en posición interna.

Cuando el no-nucleo de la construcción contiene un sustantivo, este actúa como el complemento restrictivo de un adjetivo (simple o derivado), núcleo de la construcción (24). Esto ocurre independientemente de la lengua de procedencia del compuesto.

(24) compuestos patrimoniales: pelirrojo, manilargo compuestos ingleses: tax-free 'libre de impuestos'; stone-cold 'muy frío' compuestos neoclásicos: puntiforme, canceriforme compuestos de imitación culta o inglesa: maniobrado, drogodependiente

Cuando el no-núcleo de la construcción no contiene un sustantivo, este actúa como un modificador de la categoría en posición externa, sea un nombre, un verbo, etc. El núcleo de la construcción es típicamente un sufijo que da la categoría de adjetivo a la formación en su totalidad (25).

(25) quinceañero, malintencionado

left-handed 'zurdo', over-worked 'saturado'

\section{El NÚMERO DE MORFEMAS IMPORTA: LA MATERIALIZACIÓN DE LA CATEGORÍA VERBO}

En este último apartado vamos a analizar diferentes tipos de compuestos integrados por verbos (lavaplatos, en inglés, dish-washer, malinterpretar, etc.). Los verbos presentan evidentes diferencias morfológicas en estos compuestos de acuerdo a su posición en el compuesto (interna o externa) o a su lengua de procedencia (español o inglés). En este apartado vamos a relacionar dichas diferencias morfológicas con la presencia de mayor o menor estructura funcional en el compuesto. Concretamente, plantearemos que la selección de una forma flexionada como lava, pese a no admitir variación flexiva (*lavanplatos) tiene 
consecuencias interpretativas relevantes de tipo temporal y aspectual. Estas diferencias justifican que los compuestos verbonominales romances no deban considerarse únicamente la versión derivada-cero de los compuestos verbales ingleses (Di Sciullo 1991); tampoco su versión exocéntrica.

Representamos cartográficamente la estructura funcional de los verbos en un compuesto en (26):

\section{(26) ...Modo>Tiempo>verbo(pequeño) >Verbo}

La aceptación de esta estructura viene acompañada de las siguientes asunciones. En primer lugar, siguiendo a Baker (2003), consideramos que el verbo es la única categoría léxica que proyecta inherentemente un especificador. Hablar de $V$ y no de raíz puede estar justificado desde el momento que encontramos algo como lava+ platos (argumento interno). En segundo lugar, consideramos, siguiendo a Ramchand (2008), que la informacion relativa al aspecto léxico de un verbo se divide en tres proyecciones funcionales llamadas Inicio, Proceso y Resultado. Los argumentos verbales reciben diferentes interpretaciones a expensas de la proyección verbal que los alberga (se interpretan como iniciadores, experimentantes, etc.). En este trabajo asumimos la equivalencia entre $v$ pequeño e Inicio.

En español, encontramos una proyección completa de un verbo (con tiempo, modo, $v o z$, etc.) cuando aparece en posición externa de un compuesto (27)

(27) Juan hubiera sido maleducado por sus padres de cualquier manera.

Sin embargo, encontramos una proyección parcial del verbo tanto en los compuestos verbonominales - con el verbo en posición interna)- de (28a) como en los llamados compuestos sintéticos del inglés de (28b).

(28) a. lavaplatos, cumpleaños, mandamás

b. dish-washer, expert-tested, long-lasting

Hemos observado que los compuestos de la serie (28a) presentan unas propiedades distribucionales mucho más restringidas que los de la serie (28b).

La serie de (28b) admite la interpretación de los sustantivos que aparecen en el esquema como argumentos o como adjuntos de diferente tipo (agentes, instrumentos) (29a), pero la serie de (28a) solo admite la interpretación de los sustantivos como argumentos (temas) (29b), contraste ya fuera observado en Lieber (1983).

(29) a. car-driver (tema) expert-tested (agente)

Sunday-driver (adjunto temporal) hand-written (instrumental) 
b. ${ }^{*}$ pruebaexpertos, * niegapropio, * ${ }^{*}$ conduceDomingos

*escritomano, pasacalles (locativo-tema incremental)

La serie inglesa admite la aparición productiva de modificadores (30a), la española de (30b) no:

(30) a. odd-sounding name, ill-educated person

b. *nombre suenararo, ${ }^{*}$ persona educadamal mandamás, catalejo

En los compuestos sintéticos ingleses, los argumentos externos pueden recibir los mismos roles semánticos (entre ellos, el de experimentante) que los derivados en -er (o los españoles en -dor) (31a). El argumento externo de los compuestos españoles nunca admite la interpretación del argumento externo como experimentante (31b).

(31) a. radio-hater, TV-viewer como vividor, oidor

b. ${ }^{*}$ odiarradios, ${ }^{*}$ veteles, ${ }^{*}$ vivevidas

Morfológicamente, las palabras derivadas del inglés y sus compuestos sintéticos no difieren en lo que respecta a la stem verbal sobre la que se forman (32a), mientras que las palabras derivadas del español y los compuestos del esquema verbonominal sí difieren (32b):

(32) a. washing machine, dish-washer, I am washing

b. cubrir, cubridor, pero cubretetera cumplir, cumplido, pero cumpleaños

En (32) se señala la (ampliamente notada) particularidad de que los compuestos verbonominales españolas se forman con una forma flexionada de presente de indicativo. En nuestra aproximación, esto deja de ser un capricho morfológico, al reflejar propiedades estructurales relevantes del esquema romance. Así, la interpretación aspectual de lavaplatos solo puede ser activa y nunca progresiva. La interpretación progresiva se podría haber obtenido en el compuesto romance si la forma verbal fuera un infinitivo (el lavar los platos...), pero no lo es.

Los contrastes de (31) nos obligan a plantearnos la siguiente pregunta: ¿Por qué la interpretación de los argumentos externos como agentes o instrumentos no está vinculada a la aparición de un afijo mientras que la de experimentantes sí? La respuesta que proponemos confía en la hipótesis siguiente: las formas verbales flexionadas del compuesto romance se insertan en una posición más alta de la cartografía del verbo que los temas verbales que aparecen (sin flexionar) en un derivado.

La forma verbal lava de lavaplatos se inserta sobre v pequeño o Inicio. Ello determina que los argumentos externos se interpreten con alguno de los roles temáticos vinculados 
a Inicio (agente, causa, instrumento) y nunca como experimentantes. Experimentantes e iniciadores no son variantes de un papel temático para una posición, sino papeles vinculados a proyecciones distintas (33):

(33) Juan molesta a sus vecinos

Causa Experimentante

Los compuestos sintéticos del inglés han de esperar hasta la inserción del afijo para la identificación de $\mathrm{v}$ pequeño. A expensas del valor activo (-er), progresivo (-ing) o pasivo $(-e d)$ de dicho sufijo variará, no solo la interpretación del argumento externo, sino también la interpretación del sustantivo interno como tema, agente, instrumento, etc. Ello nos permite explicar los contrastes interpretativos de (29). Dejamos pendiente de explicación el contraste relativo a la aparición productiva de adjuntos (30), aunque posiblemente este hecho esté relacionado con diferencias de tipo general entre los patrones compositivos de ambas lenguas.

\section{Obras Citadas}

Ackema, Peter y Neeleman, Ad. 2004. Beyond Morphology: Interface Conditions on Word Formation. Oxford: OUP.

Baker, Mark. 2003. Lexical Categories: Verbs, Nouns and Adjectives. Cambridge: CUP.

Benveniste, Émile. 1966. "Formes nouvelles de la composition nominale". Bulletin de la Société Linguistique de Paris 61: 82-95.

Bobaljik, Jonathan David y Thráinsson, Höskuldur. 1998. “Two Heads Aren't Always Better Than One". Syntax 1.1: 37-71.

Borer, Hagit. 2013. Structuring Sense: Taking form. Vol 3. Oxford: OUP. . 2005. Structuring Sense. In Name Only. Vol. 1. Oxford: OUP.

Chomsky, Noam. 1970. "Remarks on Nominalization". Studies on Semantics in Generative Grammar. The Hague: Mouton. 11-61.

Cinque, Guglielmo. 2014. "The semantic classification of adjectives. A view from syntax". Studies in Chinese Linguistics 35: 3-32.

Croft, William. 1991. Syntactic categories and grammatical relations. Chicago: UCP.

DiSciullo, Anna María. 1991. "On the structure of Deverbal Compounds". Working Papers in Linguistics [Venice] 3: 72-112.

Fábregas, Antonio. 2005. La definición de la categoría gramatical en una morfología orientada sintácticamente: Nombres y adjetivos. Madrid: UAM.

Fábregas, Antonio y Pérez Jiménez, Isabel. 2008. "Gender agreement on adverbs in Spanish". Journal of Portuguese Linguistics 7, 2: 25-45.

García Page, Mario. 2011. "Hombre clave, hombre rana, ¿un mismo fenómeno?”. Verba 38: $127-170$. 
Gil Laforga, Irene. 2014. La interacción de los componentes gramaticales en la formación de palabras. Adjetivos posesivos derivados y compuestos. Madrid: UAM.

Givon, Thomas. 1979. On Understanding Grammar. New York: Academic Press.

Harley, Heidi. 2009. "Compounding in Distributed Morphology". En Rochelle Lieber y Pavol Štekauer, eds., The Oxford Handbook of Compounding. Oxford: Oxford University Press, 129-144.

Hinzen, Wolfram. 2008. "Prospects for an explanatory theory of semantics". Biolinguistics 2, 4: 348-363.

Lieber, Rochelle. 1983. "Argument Linking and Compounds in English". Linguistic Inquiry 14, 2: 251-285.

\section{UCP.}

. 1992. Deconstructing Morphology: Word Formation in Syntactic Theory. Chicago:

Moyna, Maria Irene. 2011. Compound Words in Spanish: Theory and History. Amsterdam: John Benjamins.

Rainer, Franz y Varela, Soledad. 1992. "Compounding in Spanish”. Rivista di Linguistica 4, 1: 117-142.

Ramchand, Gilliam. 2008. Verb meaning and the lexicon: First phase syntax. Cambridge: CUP.

Rizzi, Luigi. 1997. "The Fine Structure of the Left Periphery”. En Liliane Haegeman, ed., Elements of Grammar. Kluwer: Dordrecht. 281-337.

Scalise, Sergio, Antonio Fábregas y Francesca Forza. 2009. "Exocentricity in Compounding”. Gengo Kenkyu 135: 49-84.

Starke, Michal. 2004. "On the inexistence of specifiers and the nature of heads". En Adriana Belletti, ed., Structures and beyond: The cartography of syntactic structures. Oxford: OUP. 252-268.

Val Álvaro, José Francisco. 1999. "La composición”. En Ignacio Bosque y Violeta Demonte, eds., Gramática Descriptiva de la Lengua Española. Vol. 3. Madrid: Espasa-Calpe. 4757-4842.

Varela, Soledad. 1990. "Composición nominal y estructura temática". Revista Española de Lingüistica 20, 1: 55-81. 